\title{
2 Vektoralgebra
}

\subsection{Kosinussatz}

Verallgemeinerter Kosinussatz:

$$
|\vec{a}-\vec{b}|^{2}=|\vec{a}|^{2}+|\vec{b}|^{2}-2|\vec{a}||\vec{b}| \cos \gamma
$$

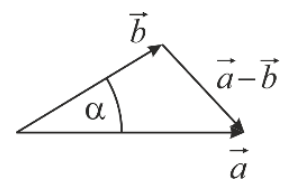

\subsection{Spatprodukt}

Spatprodukt dreier Vektoren:

$$
\begin{aligned}
\langle\vec{a}, \vec{b}, \vec{c}\rangle & =\operatorname{det}(\vec{a}, \vec{b}, \vec{c}) \\
& =\left|\begin{array}{lll}
a_{1} & b_{1} & c_{1} \\
a_{2} & b_{2} & c_{2} \\
a_{3} & b_{3} & c_{3}
\end{array}\right|=\vec{a} \cdot(\vec{b} \times \vec{c})
\end{aligned}
$$

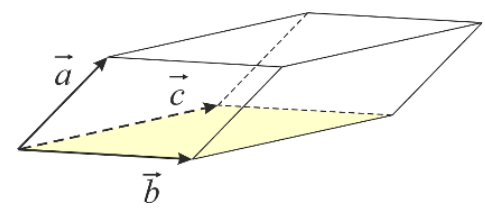

Hinweise:

- Spatprodukt liefert (orientiertes) Volumen des aufgespannten Parallelepipeds.

- Ergebnis kann auch negativ sein (Selbstdurchdringung bei Finiten Elementen).

- Zyklische Vertauschung ist möglich: $\vec{a} \cdot(\vec{b} \times \vec{c})=\vec{b} \cdot(\vec{c} \times \vec{a})=\vec{c} \cdot(\vec{a} \times \vec{b})$

\subsection{Abstandsvektoren}

Veranschaulichung des Abstandsvektors $\vec{d}$ zwischen einem Punkt $P$ und einer Geraden $g$ sowie zwischen zwei Geraden $g_{1}$ und $g_{2}$ :
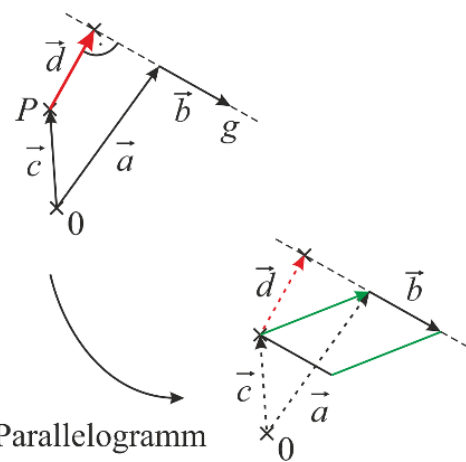
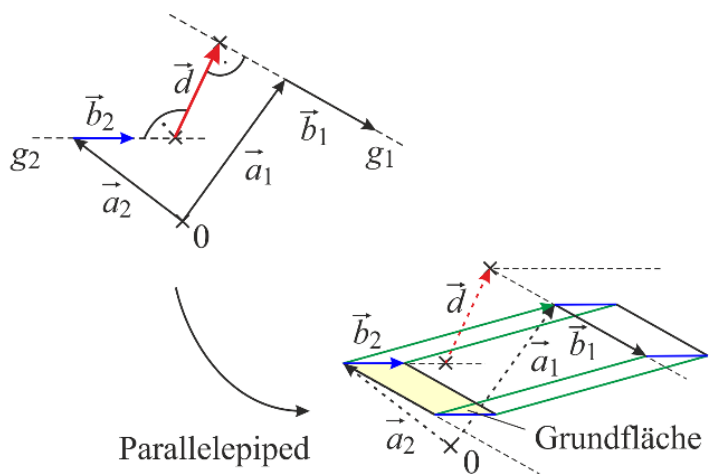